\title{
The prevention of spread of methicillin resistant Staphylococcus aureus in a spinal injuries centre
}

\author{
F C M Pick RGN RM RCNT, ${ }^{1}$ M Rose SRN,${ }^{2}$ D Wang MD Visiting Professor, \\ B P Gardner MA MRCP (UK) FRCS, ${ }^{2}$ A P Gillett MRCP FRCPath ${ }^{3}$
}

\author{
${ }^{1}$ Infection Control, Stoke Mandeville Hospital, Aylesbury, Bucks, ${ }^{2}$ National Spinal \\ Injuries Centre, Stoke Mandeville Hospital, Aylesbury, Bucks, ${ }^{3}$ Department of \\ Microbiology, Stoke Mandeville Hospital, Aylesbury, Bucks, England
}

\begin{abstract}
The National Spinal Injuries Centre (NSIC) is a tertiary referral centre. It accepts most of its patients from other hospitals in the UK and overseas. The severity of injury, the presence of a tracheostomy, urinary catheter and pressure sores predisposes this group of patients to colonisation or infection with Methicillin resistant Staphylococcus aureus (MRSA). The NSIC uses simple but strict protocols for hygiene, screening for MRSA, and source isolation of known or suspected MRSA carriers in single room accommodation to control the spread of MRSA in the centre. A retrospective search of microbiology and patient records revealed that in 4 years there had been 24 admissions with MRSA, with a total of 1421 isolation days. There was only one outbreak of MRSA. This involved three patients. Hygiene, screening of potential MRSA carriers together with single room isolation can limit the spread of MRSA.
\end{abstract}

Keywords: methicillin resistant Staphylococcus aureus (MRSA); incidence; prevalence; screening; source isolation; spinal injuries.

\section{Introduction}

Methicillin resistant Staphylococcus aureus (MRSA) is a world-wide problem, with particular relevance to tertiary referral centres. ${ }^{1}$ Physical dependency, prolonged hospital stay, urinary catheters, pressure sores and other chronic wounds are predisposing factors for colonisation or infection with MRSA. ${ }^{2}$ All are recognised complications of spinal injured patients. Long-stay patients with MRSA provide persistent reservoirs allowing the spread to others. ${ }^{3} \mathrm{~A}$ literature search revealed no references specific to MRSA in spinal injuries.

Clinical, ethical, financial and increasingly, medico-legal reasons exist for preventing the spread of infection. Preventing the spread of MRSA is less costly than controlling an outbreak. ${ }^{4}$

Other hospitals have found single room isolation inadequate for preventing the

Correspondence: Mrs F C M Pick, Stoke Mandeville Hospital, Aylesbury, Bucks. HP21 8AL, England. spread of MRSA. There have been recommendations that isolation wards should be introduced. ${ }^{5}$

This study examines the role of simple but strict protocols for hygiene, screening and source isolation in the limitation of the spread of MRSA.

\section{Background policies and practices}

The National Spinal Injuries Centre (NSIC) is a 120 bedded unit on a District General Hospital site with which it shares support facilities. For the past 10 years the centre has had a policy consistent with national guidelines $^{6}$ for the prevention of spread of MRSA. At no time has MRSA been an endemic problem in any part of the hospital. Patients admitted from areas not known to be free from MRSA are screened prior to or on admission. The sites screened are nose, axillae, perineum, urine (if the patient is catheterised) and all altered skin sites such as tracheostomies, wounds, pressure sores and eczematous lesions. Patients suspected 
of, or screening positive for MRSA, are nursed in a single room using strict source isolation techniques. ${ }^{6}$ In particular the door is kept shut and protective clothing consisting of disposable gloves and plastic aprons are worn for contact with the patient and the immediate environment. Occasionally impervious long sleeved gowns are used for nursing patients with extensive sores.

Attendants always wash their hands on leaving the room using liquid soap or an alcohol hand rub. Non-disposable equipment is disinfected, using heat or a clear soluble phenolic solution prior to use by another patient. The healing of acute and chronic wounds is promoted.

\section{Patients infected or colonised with MRSA}

The patient's medical treatment is not compromised as essential visits to $\mathrm{x}$-ray and surgery are conducted using the appropriate precautions. $^{6}$ Rehabilitation may be restricted as patients screening positive for MRSA do not visit physiotherapy, occupational therapy, or hydrotherapy departments. Isolation precautions are withdrawn when the patient has three consecutive negative screens after discontinuing systemic and local antibiotic therapy. Regular screening for MRSA is continued during the patients hospital stay and on subsequent admissions.

MRSA organisms are sent to the Staphylococcal Reference Laboratory at Colindale for phage typing.

\section{Study methods}

Microbiology and infection control records were searched to identify patients who initially screened positive for MRSA from October 1987 to September 1991.

These patients' case notes and microbiological records were reviewed retrospectively to determine their predisposition to acquiring MRSA, the place of acquisition (NSIC or other) and clinical and microbiological outcome. Results of phage typing and antibiotic sensitivity patterns were examined for evidence of cross-infection.

\section{Definitions}

(1) Infected. Patients were considered infected if there was visible inflammation at a site shown positive for MRSA, or bacteraemia.

(2) Colonised. Patients without the above criteria were considered to be colonised with MRSA.

\section{Results}

Twenty-four admissions to the NSIC (23 patients of whom 20 were male and three female) screened positive for MRSA during the study period. This represented $0.87 \%$ of all admissions. The incidence of MRSA admissions in the rest of the hospital was $0.02 \%$. Twenty patients screened positive for MRSA prior to, or on admission. Three patients acquired MRSA in the NSIC. Of the 20 admitted with MRSA, 14 were from overseas: Australia, Egypt, France, Greece, Ireland, Israel, Portugal, Spain and the United States of America; six were from London and the South of England.

The incidence rate (Fig 1) increased during the study period from two in year 1 to 11 in year 4 .

Twenty-three patients had suffered traumatic spinal cord injury and one had multiple myeloma. The level of injury was cervical in 12, thoracic in nine, and lumbar in two. Although all patients admitted to the NSIC had previously been in other hospitals, five required intensive care and eight required respiratory assistance via tracheostomy. All but one patient had pressure sores and/or a urinary catheter. Table I shows

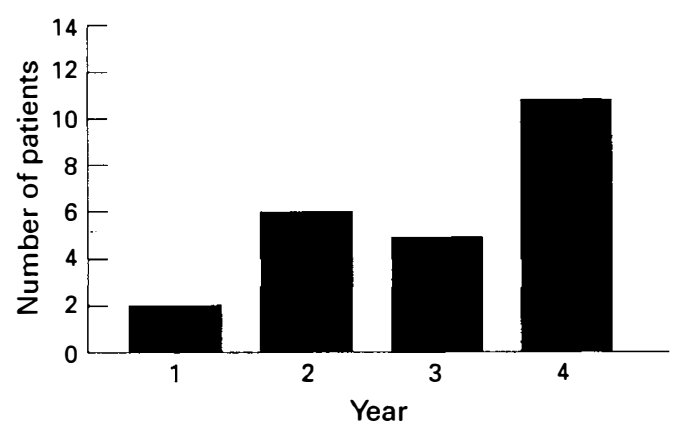

Figure 1 Incidence of MRSA by year. 
Table I Patient profiles including predisposing factors for acquiring MRSA

\begin{tabular}{lc}
\hline Patient factors & Number of patients \\
\cline { 1 - 2 } Intensive care & 5 \\
Tracheostomy & 8 \\
Pressure sore(s) & 17 \\
Urinary catheters & 20 \\
Multiple injuries & 12 \\
Cervical injury & 12 \\
\hline
\end{tabular}

details of patient profiles including factors predisposing to infections.

Of the 23 patients 13 were infected and 10 colonised with MRSA. Ten patients received systemic antibiotics, two in conjunction with topical mupirocin and two patients had topical mupirocin only. Ten patients had no records of antibiotic therapy.

On discharge, nine of the admissions still screened positive for MRSA, (four of these were short stay patients and were in the NSIC for less than 20 days), 15 screened negative. Treatment of MRSA carriage was unsuccessful while the patients had tracheostomies and/or sores. Once broken skin had epithelialised treatment was more likely to succeed.

The number of days spent in isolation ranged from 3-183, mean 59.2. Five patients were in isolation for more than 100 days and six patients less than 20 days. Total isolation days was 1421 .

Of the three patients who acquired MRSA in the NSIC, one was infected and two were colonised. All had swabs from pressure sores cultured positive for MRSA the first time in January and February 1991. Their organisms all had the same antibiograms. All were untypable. The index case was thought to be an outpatient with a matched organism. Epidemiological investigations showed the only common factor was a doctor, who, being the only link between the outpatients and the inpatients was assumed to have acted as vector.

\section{Organism profiles}

Strains of MRSA from six patients were untypable using routine and experimental phages. These included the strains from the
Table II Antibiotic resistance of tested strains

\begin{tabular}{lcc}
\hline Antibiotic & Strains tested & Strains resistant \\
\hline Penicillin & 24 & 24 \\
Methicillin & 24 & 24 \\
Erythromycin & 24 & 23 \\
Tetracycline & 24 & 19 \\
Gentamicin & 24 & 13 \\
Rifampicin & 19 & 7 \\
Mupirocin & 15 & 1 \\
Vancomycin & 21 & 0 \\
Teicoplanin & 15 & 0 \\
\hline
\end{tabular}

three patients who acquired MRSA in the NSIC. The other three untypable strains had differing antibiograms. Of the remaining strains typed, two resembled MRSA 1, the rest all had different phage types. Seventeen strains were resistant to five or more commonly tested antibiotics. Table II shows the individual antibiotics to which tested strains were resistant. All strains were resistant to penicillin and methicillin and all tested were sensitive to vancomycin and teicoplanin, most strains were resistant to erythromycin and tetracycline and over half were resistant to gentamicin. Only one of the strains tested was mupirocin resistant.

\section{Discussion}

Lengthy hospital stays for patients affected by MRSA result in a significant increase in the prevalence of MRSA. During the study period there were 1421 isolation days (3.9 years) during which a patient was in the NSIC, with MRSA acting as a potential reservoir for spread, if infection control procedures broke down. The effect on the patient of prolonged isolation is difficult to quantify. The five patients who were isolated for more than 100 days had cervical lesions and tracheostomies and/or pressure sores. Attempts at eradication with antibiotics were unsuccessful. Their rehabilitation would have been limited by their clinical condition during this period, whether they had been isolated or not. In other patients, rehabilitation was restricted until they were cleared from MRSA. It was not possible to identify if, or by what time span, hospital stay was extended. 
Although it is known that MRSA exists world-wide, it is not known whether the strains imported into the NSIC are epidemic in their country of origin, or how easily they are transmitted within a setting such as the NSIC. Would these strains have spread without isolation? Experience from other tertiary referral units suggests they do spread (personal communications from infection control nurses).

MRSA was introduced to the unit on 21 occasions in 4 years and was present for 1421 days. Only one episode of spread affecting three patients, suggests that considering the levels of nursing, medical and paramedical intervention required by the patients the control methods used were successful.

\section{Conclusions}

(1) The incidence and prevalence of MRSA being introduced to a tertiary referral centre may be higher than in an associated district general hospital.

(2) Persistence of MRSA in tracheostomies and pressure sores is a significant problem in patients with spinal injuries.

(3) There was no endemic strain of MRSA present in the NSIC during the study period as evinced by differing phage types and antibiograms, together with no sporadic cases of MRSA carriers.

(4) Simple but strict protocols for screening, hygiene and isolation can limit the spread of MRSA in a spinal injuries centre.

\section{References}

1 Hayley RW, Hightower AW, Khabbaz RF et al (1982) The emergence of Methicillin resistant Staphylococcus aureus in United States hospitals. Anals Int Med 97: 296-308.

2 Cooke EM, Casewell MW, Emmerson M et al (1986) Methicillin resistant Staphylococcus aureus in the U.K. and Ireland. A questionnaire survey. J Hosp Infect 8: 143-148.

3 Coleman DC, Cafferkey C, Keane CT et al (1986) Mechanisms of pathogenicity of multi-resistant Staphylococcus aureus. J Hosp Infect 7: Supp A, 29-35.

4 Webster M (1986) Control measures. Nursing Times 82: 6, 26-28.

5 Duckworth GJ, Lothian JLE, Williams JD et al (1988) Methicillin resistant Staphylococcus aureus; reports of an outbreak in a London teaching hospital. J Hosp Infect 11: 1-15.

6 Ayliffe GAJ et al (1990) Revised guidelines for the control of epidemic Methicillin resistant Staphylococcus aureus. Working Party report. J Hosp Infect 16: 351-377. 